\title{
Experimental analysis of amblyopia and strabismus
}

\author{
COLIN BLAKEMORE AND RIGHARD G. VAN SLUYTERS \\ The Physiological Laboratory, Cambridge
}

In the past few years physiological experiments have brought us a little closer to an understanding of some forms of developmental amblyopia-deficits of visual acuity of central, not retinal, origin, which are not correctable by optical means. While amblyopia has become a catch-all term that covers the symptoms of a multitude of disorders, there are certain stereotyped experimental procedures that invariably lead to gross disturbances in the visual system. In this paper we shall describe a few of these experiments and attempt to relate them to some of the clinical origins of amblyopia in man.

The cat has been the subject of a great deal of research on the development of the visual system, though it is clear that most of the observations, with some minor differences, also apply to monkeys (see von Noorden, 1974). By extrapolation, the situation is probably similar in man.

Cats, monkeys, and man are all highly binocular animals. They have roughly the same amount of binocular overlap of the two visual fields, and their eyes are extremely mobile, showing disjunctive as well as conjugate movements. There is now behavioural evidence that both cats (Fox and Blake, I97I) and monkeys (Bough, 1970) normally have stereoscopic vision, being able to discriminate the relative distances of objects solely on the basis of the retinal disparity of their images. Indeed, from neurophysiological experiments there is now a fair understanding of the actual neural mechanism of stereopsis.

\section{Organization of the visual pathway and the basis of binocular vision}

Analysis of retinal disparity seems to demand a convergence of information from the two eyes at some point in the visual pathway. The opportunity for binocular input to individual neurones exists, of course, at any point central to the partial decussation of optic nerve fibres in the optic chiasm, but at the first synaptic relay in the lateral geniculate nucleus very few cells can actually be excited by visual stimuli shown to either eye. The nucleus is, in fact, divided into distinct laminae, each with principal input from one eye or the other (Kaas, Guillery, and Allman, 1972).

However, massive binocular interaction certainly occurs at the termination of geniculate cell axons in the primary visual cortex (area I 7). Most neurones of the visual cortex have excitatory input from both eyes and each cell has a specific optimal stimulus or trigger feature that will cause it to respond. The required stimulus is almost always a dark or light bar or a black-white edge at a particular orientation (Hubel and Wiesel, 1962, 1968). Different cells respond to different orientations but the preferred orientation is always quite similar in the two eyes (Blakemore, Fiorentini, and Maffei, 1972).

These binocular cortical cells appear to play a part in binocular depth discrimination. The two receptive fields (the areas of the two retinae from which a cortical cell gathers input) are not always on exactly corresponding points. There is considerable variation in 
the retinal disparity of the two receptive fields, over a total range of several degrees (Barlow, Blakemore, and Pettigrew, I967; Joshua and Bishop, I970; Hubel and Wiesel, 1970a). What is more, neurones often demonstrate a much stronger response when a target of the correct orientation appears in the correct position on both receptive fields, than when only one eye is open or when both eyes are open but the disparity of the two images is even slightly inappropriate (Barlow and others, 1967; Joshua and Bishop, 1970). This is a system, then, of disparity detecting cells: each neurone responds best when an object of the appropriate shape appears at a particular distance from the animal (for a fixed eye position). The integrity of this delicate and beautiful mechanism depends on a period of intensive development early in the animal's life. Disturbance of the visual input during this crucial phase leads to a level of disorganization of the visual cortex quite sufficient to explain the appearance of amblyopia and the loss of binocular visual function.

\section{"Deprivation amblyopia"}

If a kitten is reared in total darkness or, more conveniently, if it is binocularly deprived by suturing closed the lids of both eyes, it subsequently has gross deficits in visuomotor coordination, almost to the point of total blindness, with slow and only partial recovery (Ganz, Hirsch, and Tieman, I972). In such an animal the visual cortex is also abnormal. Although the majority of cells are still binocularly driven, many of them respond equally

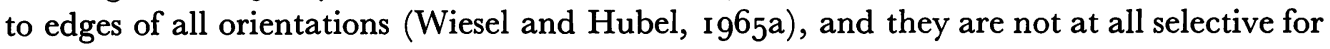
the retinal disparity of stimuli: some workers report that in such binocularly deprived cats there are no truly orientation selective cells (Barlow and Pettigrew, 1971). Here then is a fairly close correspondence between the animal's behavioural ability and the organization of its cortical neurones.

Deprivation of experience in only one eye has even more dramatic consequences. In a monocularly deprived kitten, cortical neurones have normal orientation selective properties but virtually every cell can be driven only through the visually experienced eye (Wiesel and Hubel, 1965a). Such a monocularly deprived animal has good vision through the experienced eye, but appears to be almost totally blind when forced to use its deprived eye. Clinical experience shows that, in human infants also, degradation of the visual image in one eye is a particularly potent cause of amblyopia.

Fig. I shows a series of histograms that illustrate the extent to which cortical cells can be influenced by one eye or the other. The responses of cells are tested through both eyes in turn and the cell is classified into one of seven ocular dominance groups (Hubel and Wiesel, I962):

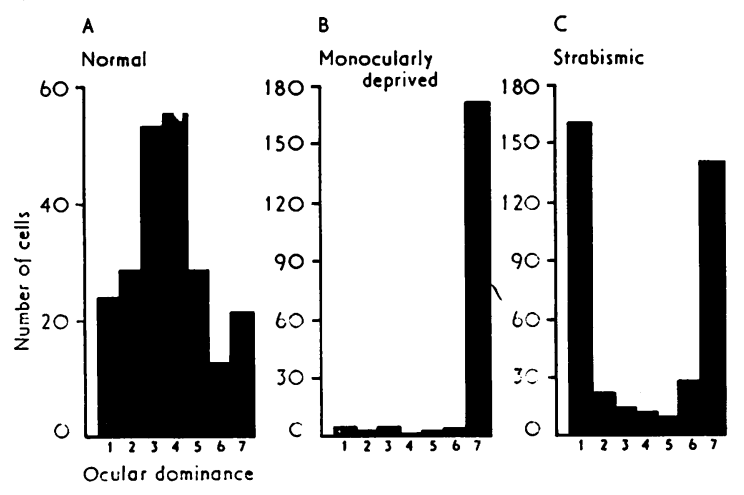

FIG. I Ocular dominance histograms for samples of cortical cells (see text for an explanation of the categories)

A. 224 cells from normal cats (Hubel and Wiesel, I962)

B. I84 cells from kittens reared with the contralateral eye deprived (Wiesel and Hubel, 1965a)

C. 384 cells from kittens with artificial strabismus (Hubel and Wiesel, 1965) 
(r) Monocular cells driven only by the eye contralateral to the hemisphere containing the cell.

(2) Binocular cells much more strongly driven by the contralateral than the ipsilateral eye.

(3) Cells driven slightly more strongly by the contralateral than the ipsilateral eye.

(4) Both eyes equal.

(5) Ispilateral slightly stronger than contralateral.

(6) Ipsilateral much stronger than contralateral.

(7) Monocular cells driven only by the ipsilateral eye.

Fig. I A shows a large sample of cells from normal adult cats while Fig. I B illustrates the results from kittens deprived of visual experience through the contralateral eye. In the latter animals, scarcely any cells are influenced by visual stimuli presented to the deprived (contralateral) retina.

These disastrous effects of uniocular deprivation in kittens occur only if one eye is occluded at some time between about 3 weeks and 3 months of age (Hubel and Wiesel, I $97 \mathrm{Ob}$ ), and they cannot be reversed by a prolonged period of binocular vision after the initial deprivation. However, a procedure of "cross-patching" (suturing the lids of the experienced eye after the deprived eye has been re-opened) can lead to a total switch-over in ocular dominance, so that all cells are driven only by the initially deprived eye (Blakemore and Van Sluyters, 1974). This reversal again occurs only if the cross-patching takes place within the 3-week to 3-month "sensitive period". A judicious choice of the timing and duration of cross-patching can produce an animal with equal numbers of cortical cells driven by the two eyes, but very few binocular neurones. Such an animal has reasonable visual ability through both eyes but presumably is deficient in stereopsis. So, in amblyopic humans, cross-patching may be expected to improve the acuity of the amblyopic eye without improving stereoscopic vision.

\section{"Strabismic amblyopia"}

One of the commonest forms of amblyopia in humans is the reduction of acuity (as well as stereoscopic vision) after early strabismus. No doubt there are many causes of strabismus, but some squints are probably congenital and the ocular misalignment is secondary to a gross genetic abnormality in the visual pathway. In the Siamese cat, which often exhibits an enormous esotropia, the lamination of the lateral geniculate nucleus, the retinotopic organization of the visual cortex, and the percentage of binocular cortical cells are all highly abnormal (Hubel and Wiesel, r97I).

On the other hand, the surgical induction of strabismus, by section of the medial rectus muscle, in an otherwise normal kitten, also has considerable effect on the visual cortex (Fig. I G). Very few cells are binocularly driven and there are separate populations of neurones responding through one eye or the other (Hubel and Wiesel, I965). There is even some evidence that the strabismic eye dominates a smaller number of cortical cells than the unoperated eye (Wickelgren-Gordon, 1972). Here, then, is a good correlation with "amblyopia ex anopsia" and the lack of stereopsis experienced by strabismic humans.

It seems from all these experiments that the necessary condition for the maintenance of normal binocularity in the visual cortex is simultaneous congruent stimulation through both eyes. Almost certainly, then, the discrepancies in the two retinal images caused by anisometropia, aniseikonia, and squint can cause a loss of binocularity in the visual cortex of human infants.

"Meridional amblyopia"

Discrepancy between the two eyes is not the only condition that leads to reorganization of 
the visual cortex. There is now evidence that the visual system simply lacks the ability to process information that it has not experienced early in life. If a kitten is reared in such a way that its entire visual experience is limited to an array of black-white vertical edges, its cortex ultimately contains no neurones responding optimally to horizontal lines (Blakemore and Cooper, 1970; Hirsch and Spinelli, 1971) and the kitten has a specific reduction in visual acuity for horizontal patterns (Muir and Mitchell, I973). This environmental modification of orientation selectivity can also take place only during the 3 -week to 3 -month sensitive period (Blakemore, I974), and it can occur after as little as I hour of exposure to stripes (Blakemore and Mitchell, 1973).

In humans too there is a close analogue of this experimental procedure. Infants with extreme ocular astigmatism (which on average defocuses one meridian much more than the orthogonal), if they do not receive cylindrical spectacle correction early in life, are left with an uncorrectable "meridional amblyopia". Acuity for contours that were originally out of focus is considerably lower than that for the best focused meridian, even when the optical error is perfectly corrected (Mitchell, Freeman, Millodot, and Haegerstrom, I973). The difference in acuity between the two principal meridians can be as much as a factor of two.

Teleologically, it would be gratifying to discover some functional value in this process of environmental modification (in the same way that the loss of binocularity after strabismus can be construed as a protection against the conflict of two inter-dependent mappings between visual and motor space). The obvious possible adaptive function is that this plasticity ensures that the powers of detection of the visual system are optimally matched to the visual environment in which the animal finds itself (or, more exactly, to the environment it lived in which it was young). However, an alternative, or additional, function comes to mind. An essential property of binocular neurones, if they are really to act as disparity detectors, is that their optimal features should be quite closely matched in the two eyes, so that they discharge very strongly only when the two similar images of a single object appear on the receptive fields. Modification of orientation selectivity early in life may be important in ensuring that binocular neurones do indeed adopt very similar preferred orientations through the two eyes (Blakemore and Van Sluyters, I974).

\section{A method of preventing some consequences of strabismus}

During the past year or so we have been following up a quite fortuitous observation that we hope may be of direct value in the clinical treatment of strabismus (Van Sluyters and Blakemore, 1973; and in preparation). We have noticed that normal kittens kept in the dark, except for regular exposure to striped patterns displayed on the inside walls of a large cylindrical enclosure, often exhibit an enormous "natural" strabismus-exotropias, esotropias, and hypertropias all being common. And yet the cortical neurones of these naturally squinting cats are quite normal in their binocularity (although the distribution of preferred orientations is, of course, strongly biased towards the orientation of the stripes in the cylinder). Two questions, then, are posed by this curious observation:

(a) Why do kittens reared in stripes develop strabismus?

(b) Why does this strabismus not lead to a loss of binocularity in the visual cortex?

In fact, the first question should more properly be reframed as "Why do kittens reared in stripes not adopt correct ocular alignment?", because kittens are known to be born with gross divergence of the visual axes (Sherman, 1972). With normal visual experience the eyes gradually adopt their correct convergence angle by the age of about 6 weeks. Presumably the visual axes normally stop converging when the images of the visual world are 
correctly lined up, in rough correspondence on the two retinae, and binocular cells are therefore consistently stimulated by correlated images.

In the case of a kitten viewing an environment of repetitive vertical stripes, there is no unique convergence angle at which the visual world is "locked" in correspondence on the two retinae. Wherever the two eyes are pointing the views they gain are very similarnamely an array of vertical black-white edges. So it is hardly surprising that the final convergence angle adopted is often not appropriate. Likewise, whatever the actual alignment of the visual axes, cortical cells will constantly be stimulated simultaneously by virtually identical features through the two eyes. Therefure the necessary conditions for the maintenance of binocularity are fulfilled, despite the presence of a strabismus.

So it is the repetitive nature of the striped stimulus and the multiple ambiguity of its retinal disparity that seem to create the strabismus and to maintain cortical binocularity.

The next step was straightforward. Would a kitten with an enormous artificial strabismus, reared in a striped environment, also retain binocular cortical neurones? We have now reared a number of animals in repetitive environments after the surgical induction of strabismus and in every case binocularity has been quite normal. Figs 2 and 3 (opposite) show ocular dominance histograms from two kittens with large exotropias, that were housed in the dark with regular exposure in a striped cylinder. One kitten was exposed to vertical stripes (Fig. 2) and the other to horizontal (Fig. 3). Clearly there is no obvious loss of binocularity compared with the normal adult cat (Fig. I A), quite unlike the typical strabismic animal (Fig. I G).

Also shown in Figs 2 and 3 are polar diagrams of the distributions of preferred orientations. Each line on these diagrams represents the optimal orientation for a single cortical cell. These show the very clear bias in the distribution of preferred orientations caused by the exposure to stripes.

Now we are working to try to discover the minimum amount of exposure to repetitive texture necessary to keep up fairly normal cortical binocularity. Our hope is that we may be able to develop a simple regimen of visual treatment that could be employed with very young children exhibiting strabismus, before the age at which surgical re-alignment can be very accurately performed. If such a procedure would, as in the kitten, sustain the binocularity of cortical cells despite the strabismus, there is a possibility that, once the eyes are re-aligned by surgical or orthoptic treatment, the patient may have the chance not only of not suffering an amblyopia but also of enjoying binocular fusion and stereopsis.

R. G. Van Sluyters holds a National Institutes of Health Special Fellowship. Our research on strabismus and amblyopia in kittens has been generously supported by a Programme Grant (No. G972/463/B) from the Medical Research Council.

\section{Discussion}

VON NOORDEN It is obviously vital to know the best time to align the eyes by optical or surgical means in cases of congenital strabismus, and I have planned monkey experiments to this end. On the other hand, it is unproven that congenital strabismus is a primary motor defect and that it is only necessary to align the eyes, in order to develop binocular vision. It is also possible that we are dealing with a primary central sensory defect: an inability of cortical neurons to respond to binocular foveal visual input. A secondary deviation may then develop as a result of this congenital defect, as there is no central reward for keeping the eye straight. I do not feel, therefore, that it is reasonable to draw all the conclusions that Dr. Blakemore has drawn in the case of congenital strabismus. 


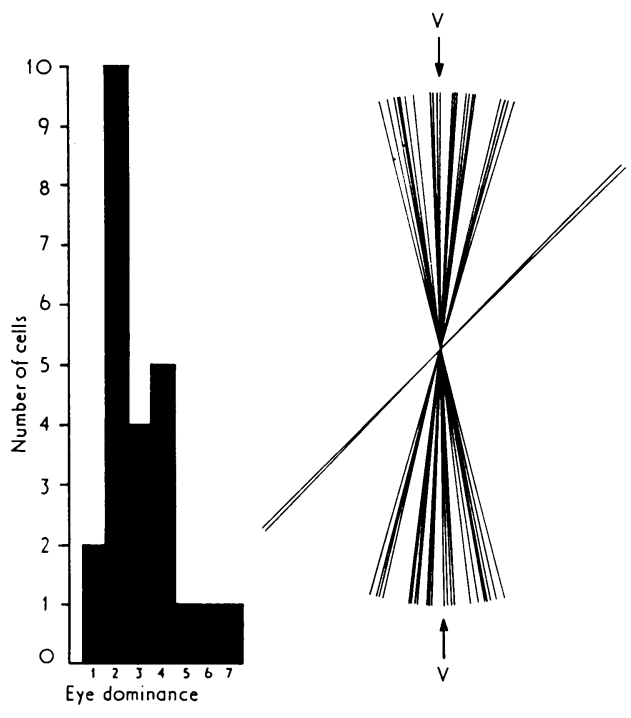

FIG. 2 Sample of 24 cortical cells from a kitten with a large artificial exotropia reared in a darkroom with regular exposure to an environment of vertical edges. On the left is a histogram of ocular dominance, on the right a polar diagram in which each line shows the preferred orientation for one neurone

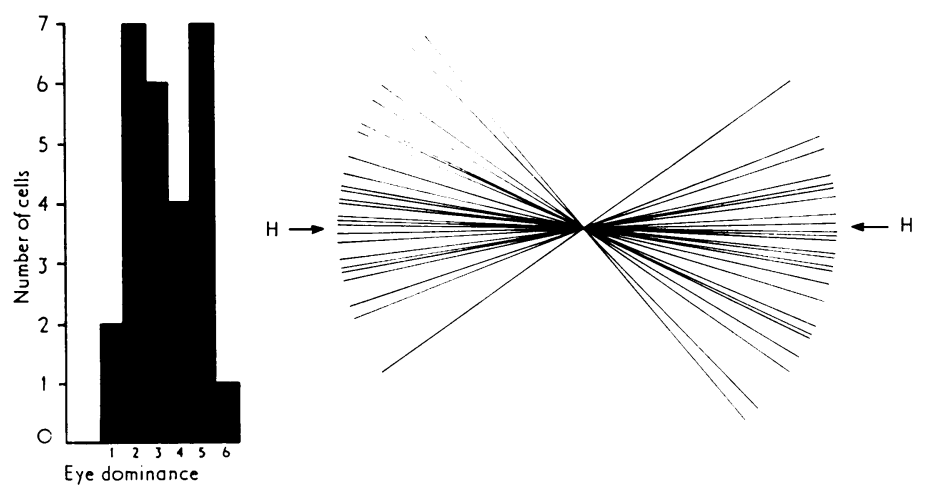

FI G. 3 A sample of 27 cortical neurones, as in Fig. 2, for a strabismic kitten exposed only to horizontal edges

BLAKEMORE The treatment we have suggested might be more appropriate in strabismus associated with a gross anisometropia or hypermetropia. However, if one keeps up the binocular stimulation of the cortex while not correcting the strabismus, then one may generate another problem-a condition far worse than amblyopia, namely the diplopia which should result. However, if there is binocularity in the cortex, then the chances of normal binocular vision after surgical correction are much, much better.

REINHART If I understood correctly, there was no correlation between loss of cortical binocularity and behavioural evidence of lowered visual acuity. Can amblyopia be produced by strabismus rather than by monocular deprivation?

VAN SLUYTERS An animal with reduced cortical binocularity as a result of a neonatal strabismus shows no apparent loss of visual acuity when tested with both eyes open or with either eye alone. Certainly the most pronounced effect that we have noticed was in the cells of the visual cortex. They lose binocularity and remain connected to either the squinting eye or the normal eye, but otherwise appear completely normal in their receptive field properties, responsiveness, and so forth. These cortical changes would suggest a loss in binocular visual function with little change in visual acuity. Unfortunately, tests of binocular visual ability are very difficclt to administer to kittens, so we have restricted our behavoural testing to a repertoire of simple visual tasks. 
BLAKEMORE Yes; our tests of behavioural function are extremely crude by the standards that are applied to humans, and they would probably only reveal a deficit so great that it would be regarded in the human as some form of blindness, not amblyopia.

HERMANN Has Dr. Blakemore made any of the animals convergent rather than divergent, as this is a situation much more commonly found in man?

BLAKEMORE I do not know of anyone who has recorded from the cortex of cats with an experimental esotropia. But there have been experiments in the Siamese cat, which often exhibits a convergent squint, known to be due to genetic rather than optical or artificially-induced factors. The cerebral organization is totally different from that found in experimental exotropic animals.

IKEDA It is extremely difficult to produce a convergent squint in the cat. We found that several muscles had to be removed completely before a permanent squint with a sign of reduction of acuity could be achieved.

WHITWELL Is a tarsorrhaphy not a very drastic form of occlusion and does not the actual trauma of the operation in some way affect the responses obtained? Will a contact lens or something rather more subtle produce the same results.

BLAKEMORE Occlusion has, in fact, been obtained by using translucent contact lenses or by suturing the nictitating membrane to the opposite area of the conjunctiva, but the results have been the same. The animals do not experience any obvious discomfort and are certainly not adversely affected by the lid-suturing operation. Our method is simple and sure, and we therefore prefer it. 